\title{
Tissue-dependent transcription of the rye centromeric histone CENH3 variants
}

\author{
Gatzkaya S.S.*, Evtushenko E.V., Vershinin A.V. \\ Institute of Molecular and Cellular Biology, SB RAS, Novosibirsk, Russia \\ *e-mail: jait@mail.ru
}

The assembly site for the kinetochore complex of active centromeres is defined by the chromosomal location of the centromeric modification of histone H3 (CENH3). The loss of CENH3 from centromeres leads to improper chromosomal segregation during cell division. In most diploid genomes, including cereals (maize and rice), in which the structure and copy number of CENH3 have been determined, CENH3 is encoded by a single gene. However, some diploid species in the tribe Triticeae have two variants of CENH3 gene. Previously we have shown the presence of two main forms of protein, $\alpha \mathrm{CENH} 3$ and $\beta \mathrm{CENH} 3$ in rye species (the genus Secale, belonging to the tribe Triticeae). In rye the average nucleotide identity between $\alpha C E N H 3$ and $\beta C E N H 3$ is $81-83 \%$, with main amino acid sequence difference in NTT domain and in $\alpha 1$-helix and loop 1 of HFD domain (CATD). Due to the presence of two main forms of the histone CENH3 instead of one, it is of great interest to study their functions. We suppose that the comparative study of the expression levels of $\alpha C E N H 3$ and $\beta C E N H 3$ in different tissues can shed light on this problem. Here we determine the expression levels of $\alpha C E N H 3$ and $\beta C E N H 3$ in various tissues of rye, Secale cereale var. 'Imperial'. The highest level of expression of both $C E N H 3$ forms was found in reproductive tissues (anther and carpel). The expression ratio of $\alpha C E N H 3$ and $\beta C E N H 3$ varies depending on the type of tissue, for example, the average expression level of $\alpha C E N H 3$ was higher than the average expression level of $\beta C E N H 3$ in leaves but they were comparable in carpels. Our results differ from the results obtained on barley, where expression level of $\beta C E N H 3$ significantly exceeded expression level of $\alpha C E N H 3$ in all studied tissues.

Acknowledgements: This research was financially supported by the Russian Fundamental Scientific Research Program (project 0310-2019-0003) and the Russian Foundation for Basic Research (grant 17-04-00748a). 